\title{
1 G-Protein signaling accelerates stem cell divisions in Drosophila males
}

2 Manashree Malpe ${ }^{1, *}$, Leon F. McSwain ${ }^{2, *}$, Karl Kudyba ${ }^{1, *}$, Chun L. $\mathrm{Ng}^{3}$, Jennie

3 Nicholson ${ }^{1}$, Maximilian Brady ${ }^{1}$, Yue Qian ${ }^{4}$, Vinay Choksi ${ }^{5}$, Alicia G. Hudson ${ }^{1}$, Benjamin

4 B. Parrott ${ }^{6}$, and Cordula Schulz ${ }^{1, \#}$

5

61 Department of Cellular Biology, University of Georgia, Athens GA 30602, USA

$7{ }^{2}$ Winship Cancer Institute, Emory University, Atlanta, GA 30322, USA

$8 \quad{ }^{3}$ University of Texas Southwestern Medical Center, Dallas, TX

$9 \quad{ }^{4}$ University of North Georgia, Department of Biology, Oakwood, GA 30566

$10{ }^{5}$ School of Medicine, Duke University, Durham, NC 27708, USA

$11{ }^{6}$ Odum School of Ecology, University of Georgia, Athens, GA 30602, USA

12 * These authors contributed equally

13 \# Corresponding author: cschulz@uga.edu 


\section{Abstract}

25 Adult stem cells divide to renew the stem cell pool and replenish specialized cells that

26 are lost due to death or usage. However, little is known about the mechanisms

27 regulating how stem cells adjust to a demand for specialized cells. A failure of the stem

28 cells to respond to this demand can have serious consequences, such as tissue loss, or

29 prolonged recovery post injury.

Here, we challenge the male germline stem cells (GSCs) of Drosophila

31 melanogaster for the production of specialized cells using mating experiments. We

32 show that repeated mating reduced the sperm pool and accelerated germline stem cell

33 (GSC) divisions. The increase in GSC divisions depended on the activity of the highly

34 conserved G-proteins. Germline expression of RNA-Interference (RNA-I) constructs

35 against G-proteins or a dominant negative G-protein eliminated the increase in GSC

36 divisions in mated males. Consistent with a role for the G-proteins in the regulation of

37 GSC divisions, RNA-i against seven out of 35 G-protein coupled receptors (GPCRs)

38 within the germline cells also eliminated the capability of males to accelerate their GSC

39 divisions in response to mating. Our data show that GSCs are receptive to GPCR

40 stimulus, potentially through a network of interactions among multiple signaling

41 pathways.

42

\section{Introduction}

44 Metazoan tissues undergo homeostasis wherein stem cells divide and their daughter

45 cells proliferate and differentiate to replace lost cells. The human hematopoietic stem

46 cells, for example, renew a remarkable number of about one trillion blood cells per day 
1,2. Stem cells have to maintain a baseline mitotic activity for the production of daughter

48 cells that account for the daily turnover of differentiated cells. However, whether stem

49 cells can modulate their mitotic activity in response to demands that challenge the

50 system is not fully explored. In some instances, stem cells respond to physiological

51 cues; for example, murine hematopoietic stem cells divide more frequently during

52 pregnancy due to increased oestrogen levels ${ }^{3}$. In Drosophila melanogaster, intestinal

53 stem cells initiate extra cell divisions upon ablation of differentiated gut cells. Drosophila

54 GSCs modulate their mitotic activity in response to environmental conditions, such as

55 nutrient availability and temperature ${ }^{4-7}$.

Drosophila is an excellent model for identifying the molecules and mechanisms

57 that regulate and fine-tune tissue homeostasis. A plethora of genetic tools are available

58 for manipulating and monitoring dividing adult stem cells in Drosophila. The small size

59 of the fly, the short generation cycle, and the fairly low costs covering their maintenance

60 allow for high throughput screens. Here, we subjected several thousand male and

61 several million virgin female flies to mating experiments, a task challenging to perform

62 with vertebrate model organisms. We discovered that repeated mating caused a

63 reproducible and significant increase in GSC division frequency in Drosophila wild-type

$64(w t)$ males. Our analysis revealed that this response to mating was dependent on the

65 activity G-proteins. Impairing G-protein activity from the germline cells eliminated the

66 ability of the GSCs to increase their division frequency in response to mating.

67 G-proteins are highly conserved molecules that associate with GPCRs. GPCRs

68 constitute a large family of cell surface receptors that mediate the cell's response to a

69 wide range of external stimuli, including odors, pheromones, hormones, and 
neurotransmitters. Loss of GPCR signaling affects countless developmental and neural

71 processes in humans, as well as vertebrate and invertebrate model organisms ${ }^{8-10}$. Here

72 we show that reducing the expression of seven out of 35 GPCRs via RNA-i from the

73 germline cells eliminated the capability of males to accelerate their GSC divisions when

74 mated. These were the Serotonin (5-HT) Receptors 1A, 1B and 7, Metuselah (Mth),

75 Metuselah-like5 (Mth-I5), Octopamineß2-Receptor (Octß2R), and a predicted GPCR

76 encoded by CG12290.

A role for any of these GPCRs in regulating GSC division frequency is novel. No

78 previous study has identified any functional role for Mth-15 or CG12290. Serotonin,

79 Octopamine, and Mth signaling play opposing roles in life-span, locomotion, and sleep

$80 \quad{ }^{11-16}$. Mth signaling also regulates vesicle trafficking at the synapse, Octopamine

81 signaling regulates ovulation, and Serotonin signaling plays essential roles in memory

82 formation and learning ${ }^{17-19}$.

\section{Results}

\section{Mating increased the percentage of GSCs in mitosis}

86 As is typical for many stem cells, the Drosophila GSCs are found in a specific cellular

87 microenvironment. They are located at the tip of the gonad, where they are attached to

88 somatic hub cells (Figure 1A, A'). Upon GSC division, one of the daughter cells, called

89 gonialblast, undergoes four rounds of stem cell daughter characteristic transit amplifying

90 divisions, resulting in 16 spermatogonia. Subsequently, spermatogonia enter a tissue-

91 specific differentiation process. They grow in size, undergo the two rounds of meiosis,

92 and develop through extensive morphological changes into elongated spermatids ${ }^{20}$. 
93 According to this tightly controlled homeostasis program, each GSC division can only

94 produce 64 spermatids (Figure 1A). Thus, an increase in sperm production is reliant on

95 the GSCs.

96 We investigate division frequency using an established immuno-fluorescence

97 protocol $^{7}$. In this approach, Vasa-positive GSCs are identified based on their position

98 adjacent to FasciclinllI (FasllI)-positive hub cells (Figure 1A'). The percentage of GSCs

99 in mitosis, the $\mathrm{M}$-phase index (MI), is investigated by staining against phosphorylated

100 Histone-H3 (pHH3). The MI of the GSCs $\left(\mathrm{Ml}^{\mathrm{GSC}}\right)$ is calculated by dividing the number of

101 pHH3-positive GSCs by the total number of GSCs.

102

To investigate if stem cells can modulate their division frequency in response to a

103 demand for specialized cells, we challenged Drosophila males in mating experiments.

104 For each experiment, 80-100 males were exposed individually to virgin females. An

105 equal number of male siblings were each kept in solitude and served as the non-mated

106 controls. To keep experimental variation to a minimum, we employed a three-day

107 mating protocol for all experiments, kept the animals under the same conditions,

108 dissected the testes at the same time of the same day, and dissected experimental

109 groups in tandem. Using wt males, we obtained robust and reproducible increases in

$110 \mathrm{MI}^{\mathrm{GSC}}$ in response to mating. The box-plot in Figure 1B shows the observed difference

111 in $\mathrm{MI}^{\mathrm{GSC}}$ between mated and non-mated populations of isogenized wt, Oregon $R(O R)$,

112 males from 17 independent mating experiments. Interestingly, we observed variability in

$113 \mathrm{MI}^{\mathrm{GSC}}$ among males of each condition. The $\mathrm{MI}^{\mathrm{GSC}}$ of non-mated males ranged from six

114 to nine percent, with a median at seven percent. The $\mathrm{MI}^{\mathrm{GSC}}$ of mated males ranged from

11511 to 18 percent, with a median at 16.5 percent. We hypothesize that this variability in 
$116 \mathrm{MI}^{\mathrm{GSC}}$ within each condition is due to naturally occurring physiological differences within

117 the flies. Likewise, the increase in $\mathrm{MI}^{\mathrm{GSC}}$ in response to mating varied among the

118 different experiments, but, in each of the experiments, the increase was biologically and

119 statistically significant.

120 We next investigated if only a few males within a population contributed to the

121 increase in $\mathrm{MI}^{\mathrm{GSC}}$ or whether the effect of mating is reflected by changes in the $\mathrm{MI}^{\mathrm{GSC}}$

122 across a population. These data are displayed in frequency distribution graphs (FDGs).

123 FDGs show how often a particular value is represented within a population. When the

124 distribution of the $\mathrm{MI}^{\mathrm{GSC}}$ for testes within one population of $O R$ flies was plotted, the

125 resulting FDG revealed that mated males had significantly fewer testes with an $\mathrm{MI}^{\mathrm{GSC}}$ of

126 zero and more testes with higher $\mathrm{MI}^{\mathrm{GSC}}$ compared to non-mated siblings (Figure 1C).

127 We observed the same result for another isogenized wt strain, Canton S (CS, Figure

128 1D). We conclude that mating affected the $\mathrm{MI}^{\mathrm{GSC}}$ of many males within one mated

129 population.

130 Finally, we asked how long or frequently we had to mate the males to see an

131 increase in $\mathrm{MI}^{\mathrm{GSC}}$. For this, we mated $O R$ males to varying numbers of virgin females

132 and subsequently analyzed how many of their GSCs were in mitotic division. When we

133 exposed OR males for 24 hours to one (1F, $24 \mathrm{hrs})$, two (2F, $24 \mathrm{hrs})$, or three (3F,

134 24hrs) female virgins, no significant difference in $\mathrm{MI}^{\mathrm{GSC}}$ between non-mated and mated

135 males was apparent (Figure S1A). Robust and reproducible increases were seen in OR

136 males that were exposed to three virgin females on each of two (2x3F, 48 hrs) or three

137 (3x3F, $72 \mathrm{hrs})$ days of mating (Figure S1A). We conclude that males have to mate

138 repeatedly for an increase in $\mathrm{MI}^{\mathrm{GSC}}$ to occur. The increase in $\mathrm{MI}^{\mathrm{GSC}}$ in mated males was 
139 reversible, showing that the response to mating was dynamic. Moving males back into

140 solitude after the three-day mating experiment for 48 hours (3x3F, $120 \mathrm{hrs}$ ) eliminated

141 the increase in $\mathrm{MI}^{\mathrm{GSC}}$ (Figure S1A). Control males mated for 120 hours (5x3F, $120 \mathrm{hrs}$ ),

142 in contrast, still had a significant increase in $\mathrm{MI}^{\mathrm{GSC}}$ (Figure S1A).

\section{Mating increased GSC division frequency}

145 As another measure of cell divisions, we investigated the percentage of GSCs in

146 synthesis phase (S-phase) of the cell cycle. Testes were labeled with 5-ethynyl-2'-

147 deoxyuridine (EdU) and the S-phase index of the GSCs ( $\mathrm{SI}^{\mathrm{GSC}}$ ) was calculated by

148 dividing the number of EdU-positive GSCs by the total number of GSCs. Using pulse-

149 labeling experiments, we observed that mated $O R$ males displayed significant higher

$150 \mathrm{SI}^{\mathrm{GSC}}$ compared to their non-mated siblings (Figure 1E). Together with the increase in

151 the $\mathrm{MI}^{\mathrm{GSC}}$ this suggests that mating accelerates stem cell divisions.

152 To test this hypothesis, the lengths of the cell cycle were measured using EdU

153 feeding experiments. In this approach, OR animals were fed EdU during the mating

154 experiment. We then calculated how many GSCs had been in S-phase at different time

155 points. Our EdU-incorporation experiment revealed that the number of EdU-positive

156 GSCs increased rapidly after 24 hours of feeding and reached $80 \%$ at 60 hours of

157 feeding (Figure 1F). Prolonged feeding further increased the numbers of EdU-positive

158 GSCs but this data was excluded from the study as the majority of males that were fed

159 EdU while mating had died by 72 hours of the experiment. The response curve we

160 obtained in this time-course experiment is different from the response curves reported

161 by other groups that used bromo-deoxy-uridine (BrDU) as the thymidine analog instead 
162 of EdU. For example, the non-mated males in our experiment had about $70 \%$ of EdU-

163 positive GSCs after 48 hours of feeding. A study using white $(w)$ mutant animals fed the

164 same concentration of the thymidine homologue had a steeper response curve, in which

$16585 \%$ of the GSCs were BrDU-marked after 48 hours of feeding ${ }^{21}$. Another study using

166 yellow, vermillion $(y, v)$ flies showed even steeper response curves where $100 \%$ of the

167 GSCs were BrDU-labeled after 24 hours. However, in this study, animals were fed a 30

168 times higher concentration of the thymidine homologue than used in our study ${ }^{22}$. We

169 propose that the different response curves are due to the different genetic backgrounds,

170 chemicals and doses.

171 Most importantly, mated males had significantly more EdU-positive GSCs at 36

172 and 48 hours of mating compared to their non-mated siblings (Figure 1F). This

173 experiment shows that, in mated males, more GSCs had entered S-phase of the cell

174 cycle. We conclude that mated males had accelerated GSC divisions.

175

To further investigate how mating affects the cell cycle, we employed the Fly-

176 Fucci technology in combination with the UAS-Ga4 expression system (Duffy, 2002

177 \#321)(Phelps, 1998 \#34)(Zielke, 2014 \#1122). With Fly-Fucci, the coding regions of

178 fluorescent proteins are fused to the destruction boxes of cell cycle regulators, allowing

179 the marking of different cell cycle stages. These artificial proteins are expressed under

180 control of the Yeast Upstream Activating Sequences ${ }^{23}$ (Zielke, 2014 \#1122). UAS-

181 controlled target genes can be expressed under spatial control using tissue-specific

182 Gal4-transactivators. In addition, temporal control can be applied to their expression by

183 exposing the flies to different temperatures (Phelps, 1998 \#34)(Duffy, 2002 \#321). For

184 our experiments, we used a nanos-Gal4-transactivator (NG4) with a reported 
185 expression in GSCs, gonialblasts, and spermatogonia (Van Doren, 1998 \#55). Using

186 two independent Fucci-lines, we observed that $\mathrm{MI}^{\mathrm{GSC}}$ did not significantly increase in

187 mated Fucci/NG4 males while mated controls animals (Fucci/wt) increased their MI ${ }^{\mathrm{GSC}}$

188 compared to non-mated siblings (Figure S1B). We conclude that expressing FUCCI-

189 constructs from these fly lines within the GSCs interfered with their ability to significantly

190 increase $\mathrm{MI}^{\mathrm{GSC}}$ in response to mating. One possible explanation for this could be that

191 the expression of proteins with destruction boxes could overload the cell cycle

192 machinery of male GSCs.

194 Mating reduced the sperm pool

195 To confirm that our mating experiments created a demand for sperm, we explored 196 differences in the sperm pool of the seminal vesicles between non-mated and mated

197 males. For this, we used two different transgenic constructs that label the sperm. A Don

198 Juan-Green Fluorescent Protein (DJ-GFP) reporter labels the sperm bodies and allows

199 to assess the overall amount of sperm within the seminal vesicles ${ }^{24}$. A ProtamineB-

200 GFP (Mst35B-GFP) line, on the other hand, only labels the sperm heads and can be

201 used to count the sperm within the seminal vesicles ${ }^{25}$. With each of these reporters,

202 individualized mature sperm was normally seen within the seminal vesicle of the male

203 reproductive tract.

204 According to the literature, the total number of sperm within one seminal vesicle 205 varies among different Drosophila species and among genetic backgrounds ${ }^{25-27}$. To

206 keep the genetic background consistent among our experiments, we crossed each of

207 the reporter lines to $O R$ females and used their male progeny for our mating 
208 experiments. The seminal vesicles were then analyzed at days one to three of the

209 experiment. Based on the size and the fluorescence of the seminal vesicles, we first

210 sorted them into three classes. Class 1 and class 2 seminal vesicles were completely

211 filled with GFP-positive sperm heads. However, class 1 seminal vesicles were very wide

212 (Figure 2A), while class 2 seminal vesicles were thinner (Figure 2B). Class 3 seminal

213 vesicles contained only few GFP-positive sperm heads and had areas that were not

214 filled with GFP (Figure 2C, arrows). A quantification revealed that non-mated males had

215 mostly class 1 and 2 seminal vesicles, while mated males had mostly class 3 seminal

216 vesicles. While we still detected class 1 and 2 seminal vesicles in males that had mated

217 for only one day, their numbers were severely reduced in males after two and three

218 days of mating (Figure 2D-F).

To further validate our observation that mating reduces the amount of sperm, we

220 developed an automated procedure that calculates the volume occupied by Mst35B-

221 GFP-positive sperm heads per seminal vesicle in all focal planes (voxels in Figure 2G).

222 This allowed us to investigate larger numbers of seminal vesicles compared to a

223 previously reported method, in which images through the seminal vesicles were

224 flattened and sperm heads counted by eye ${ }^{25}$. Furthermore, a computer-based

225 calculation eliminates subjective bias introduced by the investigator. Based on our

226 computer calculation, the sperm heads of mated males occupied significantly less

227 volume within the seminal vesicles than the sperm in non-mated males (Figure $2 \mathrm{G}$ ).

228 Notably, the total volume occupied by sperm became more reduced with every day of

229 mating. By days two and three of mating it ranged from 0.1 to $0.4 \times 10^{6}$ voxels per

230 seminal vesicle. The non-mated sibling controls, in contrast, maintained a large GFP- 
231 occupied volume in their seminal vesicles, with an average of $1.2 \times 10^{6}$ voxels per

232 seminal vesicle. The computer program estimated the numbers of sperm per seminal

233 vesicle of non-mated males around 2000, while males that were mated for two or three

234 days had less than 500 sperm in their seminal vesicles. As our mated males showed a

235 drastic reduction in sperm, we argue that we have created a demand for sperm.

237 Mating had no effect on GSC numbers

238 It was previously reported that females significantly increased the numbers of their

239 GSCs upon mating ${ }^{28}$. According to the literature, an adult male gonad contains up to

240 twelve GSCs per testis, but the exact number of GSCs per testes appears to vary

241 among different strains and laboratories. One study using a wt strain of males reported

242 six to ten GSCs per testis, while other studies using transgenic males in a $w$ mutant

243 genetic background reported 8.94 and 12.3 GSCs per testis, respectively ${ }^{29-31}$. Among

244 our fly lines, we found variation in GSC numbers as well. The distribution of GSCs

245 ranged from one to 14 per testis, with an average of seven GSCs per testis. Males from

246 an isogenized OR stock had the lowest average number of GSCs, having only four to

247 five GSCs per testis (Figure 3A). Males from an isogenized CS stock had an average of

248 six GSCs per testis (Figure 3B). Animals mutant for $w$ alleles, $w^{1118}$ and $w^{1}$, had on

249 average eight and seven GSCs per testis, respectively (Figures 3C, 3D). Males from a

$250 \quad v^{1}, y^{1}$ stock, which serves as the genetic background for many RNA-ilines, had the

251 highest average number of GSCs, at 11 GSCs per testis (Figure 3E). We believe that

252 the obtained GSC numbers are specific to the fly lines in our laboratory and do not

253 necessarily reflect the numbers of GSCs in fly stocks of other laboratories. 
Importantly, we did not observe a significant difference in the numbers of GSCs

255 between non-mated and mated siblings in any of these fly lines (Figure 3A-E). We

256 concluded that mating did not affect the numbers of GSCs in our fly stocks. However,

257 the observed variation in GSC numbers prompted us to perform our experiments in

258 animals from as similar genetic backgrounds as possible. All males reported in the

259 following of this manuscript carried the X-chromosome from our isogenized OR line.

260

261 The increase in MI GSC upon mating required G-protein signaling

262 Drosophila mating is a complex and genetically controlled behavior that is dependent on

263 neural circuits ${ }^{32}$. This implicates a possible neuronal control in regulating GSC divisions

264 during mating. Therefore, we wanted to focus on the type of signaling pathway

265 commonly stimulated during neural activity, G-protein signaling ${ }^{33,34}$. In a non-stimulated

266 cell, a trimeric complex of $\mathrm{G}$-proteins, $\mathrm{G}_{\alpha}, \mathrm{G}_{\beta}$, and $\mathrm{G}_{\mathrm{y}}$ is associated with classical

267 GPCRs (Figure 4A, step 1). When ligand binds to the GPCR, a guanidyl exchange

268 factor within the GPCR becomes activated that exchanges GDP for GTP in the $\mathrm{G}_{\alpha}$

269 subunit. The exchange leads to the dissociation of $G_{a}$ and the $G_{\beta / \gamma}$ complex from each

270 other and from the GPCR. Remaining attached to the membrane, $\mathrm{G}_{\alpha}$ and $\mathrm{G}_{\beta / \mathrm{y}}$ diffuse

271 along it and activate downstream signal transducers (Figure 4A, step 2) ${ }^{35,36}$. Most

272 organisms have multiple genes that encode for each of the G-protein subunits.

273 Drosophila has six $\mathrm{G}_{\alpha}$, three $\mathrm{G}_{\beta}$, and two $\mathrm{G}_{\mathrm{y}}$ proteins, yet only a few examples are

274 available in the literature associating a specific Drosophila G-protein with an upstream

275 GPCR ${ }^{35,37 .}$ 
Animals mutant for G-protein subunits are often lethal, making it problematic to

277 investigate their roles in the adult. Furthermore, studying G-protein signaling in animals

278 lacking their function throughout the whole body may could affect behavior and

279 physiology of the fly, leading to confounding effects on mating and GSC divisions.

280 Fortunately, large collections of RNA-i-lines are available that are expressed under

281 control of UAS. To reduce G-protein signaling we employed two separate nanos-Gal4-

282 transactivators (NG4), NG4-1 and NG4-2. When RNA-i against the different G-protein

283 subunit was expressed within the germline cells via NG4-1, several of the mated males

284 displayed only a weak increase in $\mathrm{MI}^{\mathrm{GSC}}$ compared to their non-mated siblings (Table

285 1). We focused on an RNA-i-line that is directed against the subunit $G_{\alpha} i$ as animals

286 expressing this construct within the germline did not show any increase in $\mathrm{MI}^{\mathrm{GSC}}$ in

287 response to mating (Table 1). For reproducibility, we conducted each of the following

288 experiments in triplicates. We used progeny from transgenic Gal4 and UAS-flies that

289 had been crossed to wt as positive controls. As expected, each population of positive

290 control males displayed a significant increase in $\mathrm{MI}^{\mathrm{GSC}}$ when mated (Figure 4B).

291 Experimental flies expressing $G_{\alpha} i-i$ via $N G 4-1$ or $N G 4-2$, however, failed to increase

$292 \mathrm{Ml}^{\mathrm{GSC}}$ (Figure 4C).

293 We next sought to validate the role for G-protein signaling in GSC division

294 frequency by an alternative approach. A dominant negative version of Drosophila $\mathrm{G}_{\mathrm{y}} 1$

$295\left(d n G_{y} 1\right)$ is available that serves as a reliable tool to abolish G-protein signaling ${ }^{38}$. Males

296 expressing dnG $_{\mathrm{Y}} 1$ via either NG4-1 or NG4-2 did not show an increase in MI ${ }^{\mathrm{GSC}}$ in

297 response to mating (Figure $3 \mathrm{C}$ ). Control $\mathrm{dnG}_{\mathrm{\gamma}} 1 / w t$ animals, on the other hand, had

298 increased $\mathrm{MI}^{\mathrm{GSC}}$ upon mating (Figure 3B). These data clearly show that signaling via G- 
299 proteins is required for the increase in $\mathrm{MI}^{\mathrm{GSC}}$. Plotting the results in FDGs confirmed that

300 mated control animals had significantly fewer testes with an $\mathrm{MI}^{\mathrm{GSC}}$ of zero and more

301 testes with higher $\mathrm{MI}^{\mathrm{GSC}}$ compared to non-mated males (Figures S3A-D), and that this

302 response to mating was eliminated in experimental males (Figures S3E-H).

In mammalian cells, three major G-protein-dependent signaling cascades have

304 been described (Figure 3A, steps 3a, b, c) ${ }^{33,39}$. For Drosophila, the literature provides

305 little information on the signaling cascades downstream of GPCRs but it is generally

306 assumed that the mammalian signal transducers are conserved in flies. To further

307 validate that an increase in $\mathrm{MI}^{\mathrm{GSC}}$ upon mating is regulated by G-protein signaling we

308 expressed RNA-i and mis-expression constructs for conserved signal transducers via

309 NG4 and found that males expressing RNA-i-lines for one of the Drosophila Protein

310 Kinase $C(P K C)$ proteins, PKC98E, for Inositol-triphosphate 3-Kinase (IP3K), and for

311 Ca2+/Calmodulin-dependent protein kinase /I (CaMKII) indeed failed to increase MI ${ }^{\mathrm{GSC}}$

312 in response to mating (Table 1).

314 RNA-i against seven distinct GPCRs blocked the increase in MI ${ }^{G S C}$ upon mating

315 To further confirm that G-protein signaling regulates the increase in $\mathrm{MI}^{\mathrm{GSC}}$ we aimed

316 towards identifying the upstream GPCRs. Next Generation Sequencing (NGS) of RNA

317 from $w t$ testis tips revealed the expression of 140 receptors, including 35 classical

318 GPCRs (Figure 5 and Table 2). The functions of many of these GPCRs have not been

319 studied yet and mutant animals are only available in rare cases. Expressing RNA-i-

320 constructs against most GPCRs in the germline had little to no effect on the ability of the

321 GSCs to increase their $\mathrm{MI}^{\mathrm{GSC}}$ in response to mating (Table 2). RNA-i against three 
322 Serotonin Receptors (5HT-1A, 5HT-1B and 5HT-7), Mth, Mth-15, Octß2R, and a

323 predicted GPCR encoded by CG12290, clearly and reproducibly eliminated this ability.

324 Animals carrying UAS-controlled RNA-i-constructs against these GPCRs (GPCR- $I$ ) were

325 crossed to wt, NG4-1 and NG4-2, and MI ${ }^{\mathrm{GSC}}$ of their progeny was investigated. Each of

326 the controls (GPCR-i/wt) increased their $\mathrm{MI}^{\mathrm{GSC}}$ when repeatedly mated to females in

327 each of the triplicate experiments (Figure 5A and Figures S3A-G). However, when the

328 GPCR-i-animals were crossed with either NG4-1 (Figure 5B and Figures S3H-N) or

329 NG4-2 (Figure 5C and Figures S3O-U) the $\mathrm{MI}^{\mathrm{GSC}}$ of their non-mated and mated progeny

330 did not significantly differ. Confirming the necessity of the GPCRs in increasing MI ${ }^{\mathrm{GSC}}$,

331 we investigated alternative RNA-i-lines. A second RNA-i-line for Mth blocked the

332 increase in $\mathrm{MI}^{\mathrm{GSC}}$ in mated males and a second RNA-i-line for $5 \mathrm{HT}-1 \mathrm{~A}$ displayed only a

333 weak response to mating (Table 3).

334 Mating success was evaluated by two criteria: visual observation and the

335 appearance of progeny. When flies were anesthetized to exchange the females for

336 fresh virgins, several copulating pairs of males and females were always observed.

337 Furthermore, 100 single females that had been exposed to males on day one of the

338 experiment were placed into one food vial each and mating success evaluated a few

339 days later by counting the percentage of vials with progeny. Most males in this study

340 sired $60-90 \%$ of the females. Specifically, each of the GPCR-i/NG4-1 males produced

341 offspring (Table S1), showing that a block in the increase in $\mathrm{MI}^{\mathrm{GSC}}$ is not caused by a

342 failure to mate but by a lack of GPCR signaling. Viable alleles of $5 \mathrm{HT}-1 \mathrm{~A}$ and $5 \mathrm{HT}-1 \mathrm{~B}$

343 were not pursued as alternative strategies because they displayed only a weak mating

344 success rate (Table S1). 
Finally, we wanted to assure that male age had no effect on the increase in

$346 \mathrm{MI}^{\mathrm{GSC}}$. We performed a time-course experiment of one, two, three, and four-week old

347 OR males. We found that mated males of all ages showed robust increases in $\mathrm{MI}^{\mathrm{GSC}}$

348 compared to their non-mated siblings (Figure S1C). We conclude that aging animals for

349 up to four weeks had little to no effect on the ability of wt GSCs to increase their MI GSC

350 in response to mating, and that the age of the transgenic animals used in this study

351 (three weeks of age at the time of testes dissection) had no impact on the obtained

352 results.

\section{Discussion}

355 Here, we show that repeated mating reduced the sperm pool and increased GSC

356 division frequency. Using highly controlled experiments, we demonstrate that mated

357 males had more GSCs in M-phase and S-phase of the cell cycle compared to non-

358 mated males. Mated males also showed faster incorporation of EdU indicating that their

359 GSCs progressed faster through the cell cycle. Our findings demonstrate that GSCs can

360 respond to a demand for sperm by accelerating their mitotic activity. Based on RNA-i

361 targeting G-proteins and a dominant negative construct against $\mathrm{G}_{\mathrm{y}} 1$, the increase in

$362 \mathrm{MI}^{\mathrm{GSC}}$ of mated males is dependent on G-protein signaling. Furthermore, signal

363 transducers predicted to act downstream of G-proteins and GPCRs predicted to act

364 upstream of G-proteins also appeared to be required for the response to mating.

365 Due to the lack of mutants and a potential interference of whole animal knock-

366 down in the behavior of the flies, we used tissue-specific expression of RNA-i-

367 constructs. It is surprising that our studies revealed potential roles for seven instead of a 
368 single GPCR in the increase of $\mathrm{MI}^{\mathrm{GSC}}$ in response to mating. A possible explanation is

369 that some of the RNA-i-lines have off-target effects. RNA-i-hairpins can cause the

370 down-regulation of unintended targets due to stretches of sequence homologies,

371 especially when long hairpins are used ${ }^{40,41}$. However, with the exception of the RNA-i-

372 line directed against 5HT-7, all lines that produced a phenotype contain second

373 generation vectors with a short, 21 nucleotide hairpin predicted to have no off-target

374 effects $^{42}$. We hypothesize that multiple GPCRs regulate the increase in MI ${ }^{\mathrm{GSC}}$ in

375 response to mating. Consistent with this, expression of second RNA-i-line directed

376 against Mth or $5 \mathrm{HT}-1 \mathrm{~A}$ interfered with the increase in $\mathrm{MI}^{\mathrm{GSC}}$ in mated males.

377 Our finding that RNA-i against several GPCRs blocked the increase in $\mathrm{MI}^{\mathrm{GSC}}$ in

378 mated males suggests a high level of complexity in the regulation of GSC divisions. In

379 the literature, increasing evidence has emerged that GPCRs can form dimers and

380 oligomers and that these physical associations have a variety of functional roles,

381 ranging from GPCR trafficking to modification of G-protein mediated signaling ${ }^{43-45}$. In $C$.

382 elegans, two Octopamine receptors, SER-3 and SER-6, additively regulate the same

383 signal transducers for food-deprived-mediated signaling. One possible explanation for

384 the non-redundant function of the two receptors was the idea that they form a functional

385 dimer ${ }^{46}$. In mammalian cells, 5-HT receptors can form homo-dimers and hetero-dimers

386 and, dependent on this, have different effects on G-protein signaling ${ }^{47-49}$. In cultured

387 fibroblast cells, for example, G-protein coupling is more efficient when both receptors

388 within a 5-HT4 homo-dimer bind to agonist instead of only one ${ }^{50}$. In cultured

389 hippocampal neurons, hetero-dimerization of 5-HT1A with 5-HT7 reduces G-protein

390 activation and decreases the opening of a potassium channel compared to 5-HT1A 
391 homo-dimers ${ }^{51}$. The formation of hetero-dimers of GPCRs with other types of receptors

392 plays a role in depression and in the response to hallucinogens in rodents ${ }^{52,53 .}$

Alternatively, or in addition to the possibility that some or all of the seven GPCRs

394 form physical complexes, a role for several distinct GPCRs in regulating GSC division

395 frequency could be explained by crosstalk among the downstream signaling cascades.

396 One signaling cascade could, for example, lead to the expression of a kinase that is

397 activated by another cascade. Similarly, one signaling cascade could open an ion

398 channel necessary for the activity of a protein within another cascade. Unfortunately,

399 the literature provides little information on Drosophila GPCR signal transduction

400 cascades and only very few mutants have been identified that affect a process

401 downstream of GPCR stimulation. Thus, it remains to be explored how stimulation of

402 the GPCRs and G-proteins increase GSC divisions.

403 The role for G-protein signaling in regulating the frequency of stem cell divisions

404 is novel. Our data suggest that the increase in $\mathrm{MI}^{\mathrm{GSC}}$ in response to mating is regulated

405 by external signals, potentially arising from the nervous system, that stimulate G-protein

406 signaling within the GSCs. Based on the nature of the GPCRs, the activating signal

407 could be Serotonin, the Mth ligand, Stunted, Octopamine, or two other, yet unknown,

408 signals that activate Mth-15, and CG12290 ${ }^{54-56}$. It will be interesting to address which of

409 these ligands are sufficient to increase $\mathrm{MI}^{\mathrm{GSC}}$, in what concentrations they act, by which

410 tissues they are released, and whether they also affect other stem cell populations.

\section{Methods}

\section{Fly husbandry}


414 Flies were raised on a standard cornmeal/agar diet and maintained in temperature-,

415 light-, and humidity-controlled incubators. Unless otherwise noted, all mutations,

416 markers, and transgenic lines are described in the Drosophila database and were

417 obtained from the Bloomington stock center (Consortium, 2003 \#132).

\section{UAS/Gal4-expression studies}

420 Two separate X; UAS-dicer, nanos-Gal4 (NG4-1 and NG4-2) fly lines were used as

421 transactivators. Females from the transactivator line or wt females were crossed with

422 males carrying target genes under the control of UAS in egg lay containers with fresh

423 apple juice-agar and yeast paste to generate either experimental or control flies. The

424 progeny were transferred into food bottles, raised to adulthood at $18^{\circ} \mathrm{C}$, males collected

425 and then shifted to $29^{\circ} \mathrm{C}$ for seven days to induce high activity of Gal4 prior to the

426 mating experiment. Note that the males were not collected as virgins as to avoid any

427 potential developmental or learning effects on our experiments.

\section{$429 \quad$ Mating experiments}

430 Unless otherwise noted, mating experiments were performed at $29^{\circ} \mathrm{C}$. Males and virgin

431 females were placed on separate apple juice-agar plates with yeast paste overnight to

432 assure they were well fed prior to their transfer into mating chambers. Single males

433 were placed into each mating slot either by themselves (non-mated) or with three virgin

434 females (mated) and the chambers closed with apple juice-agar lids with yeast paste.

435 Females were replaced by virgin females on each of the following two days and apple

436 juice-agar lids with yeast paste were replaced on a daily basis for both non-mated and 
437 mated animals. In most instances, females from the stock $X \square X, y, w, f / Y /$ shi ${ }^{\text {ts }}$ were

438 used as virgins. When raised at $29^{\circ} \mathrm{C}$, only females hatch from this stock. For fertility

439 tests, OR virgins were used. Note that $10-20 \%$ of the mated males died during the

440 experiment while only $5 \%$ of the non-mated siblings died.

441

442 Immuno-fluorescence and microscopy

443 Animals were placed on ice to immobilize them. Gonads were dissected in Tissue

444 Isolation Buffer (TIB) and collected in a $1.5 \mathrm{ml}$ tube with TIB buffer on ice for no more

445 than 30 minutes. Gonads were then fixed, followed by immuno-fluorescence staining

446 and imaging as previously described ${ }^{7}$. The mouse anti-FasciclinIII (FasIII) antibody

447 (1:10) developed by C. Goodman was obtained from the Developmental Studies

448 Hybridoma Bank, created by the NICHD of the NIH and maintained at The University of

449 lowa, Department of Biology, lowa City, IA 52242. Goat anti-Vasa antibody (1:50 to

450 1:300) was obtained from Santa Cruz Biotechnology Inc. (sc26877), anti-

451 phosphorylated Histone $\mathrm{H3}(\mathrm{pHH})$ antibodies (1:100 to 1:1000) were obtained from

452 Fisher (PA5-17869), Millipore (06-570), and Santa Cruz Biotechnology Inc. (sc8656-R).

453 Secondary Alexa 488, 568, and 647-coupled antibodies (1:1000) and Slow Fade Gold

454 embedding medium with DAPI were obtained from Life Technologies. Images were

455 taken with a Zeiss Axiophot, equipped with a digital camera, an apotome, and

456 Axiovision Rel. software. Statistical relevance was determined using the two-tailed

457 Graphpad student's t-test.

458

459 EdU-labeling experiments 
460 The EdU-labeling kit was obtained from Invitrogen and the procedure performed

461 following manufacturer's instructions. For EdU-pulse labeling experiments, animals

462 were mated as described above, and the dissected testes incubated with 10mM EdU in

463 PBS for 30 minutes at room temperature prior to fixation. For EdU-feeding experiments,

$464 O R$ males were fed $10 \mathrm{mM}$ EdU in liquid yeast provided on paper towels. These animals

465 were mated at room temperature $\left(21^{\circ} \mathrm{C}\right)$ because the paper towels easily dried out at

466 higher temperatures, causing the flies to dehydrate and die.

468 Sperm head volumetric calculations

469 In order to easily evaluate sperm numbers, we turned to computer analysis in Python.

470 By quantifying the volume of GFP signal we generated estimates to the amount of

471 sperm in each seminal vesicle. Image stacks were taken of individual seminal vesicles.

472 After masking relevant regions, each image set was normalized by mean subtraction

473 and division by the standard deviation, followed by rescaling image intensity to

474 encompass the range of the image. To remove signal noise, a median filter was applied

475 and the mask refined by Otso thresholding. We determined signal volume by hysteresis

476 thresholding. This approach initially thresholds an image at an upper limit, and then

477 expands the region by adjacent pixels satisfying the lower threshold. We set the lower

478 bound at the value generated from a triangle threshold and the upper threshold as the

479 median value above the lower limit. The number of signal voxels was calculated and

480 normalized to an expected size of a single sperm head. Our analysis utilized OpenCV

481 3.4.2, Scipy1.2.1, Scikit-image 0.14.2, Numpy 1.16.2, Matplotlib 3.0.3, Seaborn 0.9.0,

482 as well as built in Python 3.7.3 modules ${ }^{57-61 .}$ 


\section{References}

485

486 1. Dancey, J.T., Deubelbeiss, K.A., Harker, L.A. \& Finch, C.A. Neutrophil kinetics in man. J Clin Invest 58, 705-715 (1976).

488 2. Erslev, A. Production of erythrocytes, in Hematology. (ed. B.E. William WJ, Erslev AJ, Lichtman MA) 365-376 ( Mc-Graw-Hill, New York, NY; 1983).

491 3. Nakada, D. et al. Oestrogen increases haematopoietic stem-cell self-renewal in females and during pregnancy. Nature 505, 555-558 (2014).

493 4. Hsu, H.J., LaFever, L. \& Drummond-Barbosa, D. Diet controls normal and tumorous germline stem cells via insulin-dependent and -independent mechanisms in Drosophila. Dev Biol 313, 700-712 (2008).

496 5. Amcheslavsky, A., Jiang, J. \& Ip, Y.T. Tissue damage-induced intestinal stem cell division in Drosophila. Cell Stem Cell 4, 49-61 (2009).

498 6. McLeod, C.J., Wang, L., Wong, C. \& Jones, D.L. Stem cell dynamics in response to nutrient availability. Curr Biol 20, 2100-2105 (2010).

500 7. Parrott, B.B., Hudson, A., Brady, R. \& Schulz, C. Control of germline stem cell 501 division frequency--a novel, developmentally regulated role for epidermal growth 502 factor signaling. PLoS One 7, e36460 (2012).

503 8. Schoneberg, T. et al. Mutant G-protein-coupled receptors as a cause of human diseases. Pharmacol Ther 104, 173-206 (2004). 
505 9. Wettschureck, N. \& Offermanns, S. Mammalian G proteins and their cell type 506 specific functions. Physiol Rev 85, 1159-1204 (2005).

507 10. Langenhan, T. et al. Model Organisms in G Protein-Coupled Receptor Research. $508 \quad$ Mol Pharmacol 88, 596-603 (2015).

509 11. Lin, Y.J., Seroude, L. \& Benzer, S. Extended life-span and stress resistance in 510 the Drosophila mutant methuselah. Science 282, 943-946 (1998).

511 12. Selcho, M., Pauls, D., El Jundi, B., Stocker, R.F. \& Thum, A.S. The role of 512 octopamine and tyramine in Drosophila larval locomotion. J Comp Neurol 520, $513 \quad 3764-3785(2012)$.

514 13. Silva, B., Goles, N.I., Varas, R. \& Campusano, J.M. Serotonin receptors 515 expressed in Drosophila mushroom bodies differentially modulate larval $516 \quad$ locomotion. PLoS One 9, e89641 (2014).

517 14. Crocker, A. \& Sehgal, A. Octopamine regulates sleep in drosophila through protein kinase A-dependent mechanisms. J Neurosci 28, 9377-9385 (2008).

519 15. Yuan, Q., Joiner, W.J. \& Sehgal, A. A sleep-promoting role for the Drosophila serotonin receptor 1A. Curr Biol 16, 1051-1062 (2006).

521 16. Li, Y. et al. Octopamine controls starvation resistance, life span and metabolic 522 traits in Drosophila. Sci Rep 6, 35359 (2016).

523 17. Song, W. et al. Presynaptic regulation of neurotransmission in Drosophila by the 524 g protein-coupled receptor methuselah. Neuron 36, 105-119 (2002).

525 18. Lee, H.G., Seong, C.S., Kim, Y.C., Davis, R.L. \& Han, K.A. Octopamine receptor 526 OAMB is required for ovulation in Drosophila melanogaster. Dev Biol 264, 179$527 \quad 190(2003)$. 
528 19. Sitaraman, D. et al. Serotonin is necessary for place memory in Drosophila. Proc $529 \quad$ Natl Acad Sci U S A 105, 5579-5584 (2008).

530 20. Fuller, M.T. Spermatogenesis, in The development of Drosophila melanogaster, 531 Vol. 1. (ed. M. Bate, Martinez-Arias, A) 71-147 (Cold Spring Harbor Press, Cold $532 \quad$ Spring Harbor; 1993).

533 21. Wallenfang, M.R., Nayak, R. \& DiNardo, S. Dynamics of the male germline stem 534 cell population during aging of Drosophila melanogaster. Aging Cell 5, 297-304

536 22. Yang, H. \& Yamashita, Y.M. The regulated elimination of transit-amplifying cells preserves tissue homeostasis during protein starvation in Drosophila testis. Development 142, 1756-1766 (2015).

23. Abdouh, M., Albert, P.R., Drobetsky, E., Filep, J.G. \& Kouassi, E. 5-HT1Amediated promotion of mitogen-activated $\mathrm{T}$ and $\mathrm{B}$ cell survival and proliferation is associated with increased translocation of NF-kappaB to the nucleus. Brain

543 24. Santel, A., Blumer, N., Kampfer, M. \& Renkawitz-Pohl, R. Flagellar mitochondrial association of the male-specific Don Juan protein in Drosophila spermatozoa. $J$ Cell Sci 111 ( Pt 22), 3299-3309 (1998).

546 25. Tirmarche, S. et al. Drosophila protamine-like Mst35Ba and Mst35Bb are required for proper sperm nuclear morphology but are dispensable for male fertility. G3 (Bethesda) 4, 2241-2245 (2014). the Allocation of Ejaculate among Successive Mates by the Sperm-Limited Fly 

(1994).

553 27. Kubrak, O.I., Kucerova, L., Theopold, U., Nylin, S. \& Nassel, D.R.

$554 \quad$ Characterization of Reproductive Dormancy in Male Drosophila melanogaster. $555 \quad$ Front Physiol 7, 572 (2016).

556 28. Ameku, T. \& Niwa, R. Mating-Induced Increase in Germline Stem Cells via the Neuroendocrine System in Female Drosophila. PLoS Genet 12, e1006123

558 (2016).

559 29. Chen, D. et al. Gilgamesh is required for the maintenance of germline stem cells in Drosophila testis. Sci Rep 7, 5737 (2017).

561 30. Yamashita, Y.M., Jones, D.L. \& Fuller, M.T. Orientation of asymmetric stem cell division by the APC tumor suppressor and centrosome. Science 301, 1547-1550 (2003).

564 31. Sheng, X.R. \& Matunis, E. Live imaging of the Drosophila spermatogonial stem cell niche reveals novel mechanisms regulating germline stem cell output. Development 138, 3367-3376 (2011).

567 32. Manoli, D.S., Fan, P., Fraser, E.J. \& Shah, N.M. Neural control of sexually 568 dimorphic behaviors. Curr Opin Neurobiol 23, 330-338 (2013).

569 33. Geppetti, P., Veldhuis, N.A., Lieu, T. \& Bunnett, N.W. G Protein-Coupled 570 Receptors: Dynamic Machines for Signaling Pain and Itch. Neuron 88, 635-649 $571 \quad$ (2015).

572 34. Lee, D. Global and local missions of cAMP signaling in neural plasticity, learning, 573 and memory. Front Pharmacol 6, 161 (2015). 
574 35. McCudden, C.R., Hains, M.D., Kimple, R.J., Siderovski, D.P. \& Willard, F.S. G-

575 protein signaling: back to the future. Cell Mol Life Sci 62, 551-577 (2005).

576 36. Oldham, W.M. \& Hamm, H.E. Heterotrimeric G protein activation by G-protein577 coupled receptors. Nat Rev Mol Cell Biol 9, 60-71 (2008).

578 37. Boto, T., Gomez-Diaz, C. \& Alcorta, E. Expression analysis of the 3 G-protein 579 subunits, Galpha, Gbeta, and Ggamma, in the olfactory receptor organs of adult 580 Drosophila melanogaster. Chem Senses 35, 183-193 (2010).

581 38. Deshpande, G., Godishala, A. \& Schedl, P. Ggamma1, a downstream target for the hmgcr-isoprenoid biosynthetic pathway, is required for releasing the Hedgehog ligand and directing germ cell migration. PLoS Genet 5, e1000333 (2009).

39. Moolenaar, W.H. G-protein-coupled receptors, phosphoinositide hydrolysis, and cell proliferation. Cell Growth Differ 2, 359-364 (1991).

587 40. Kulkarni, M.M. et al. Evidence of off-target effects associated with long dsRNAs in Drosophila melanogaster cell-based assays. Nat Methods 3, 833-838 (2006).

589 41. Moffat, J., Reiling, J.H. \& Sabatini, D.M. Off-target effects associated with long dsRNAs in Drosophila RNAi screens. Trends Pharmacol Sci 28, 149-151 (2007).

591 42. Perkins, L.A. et al. The Transgenic RNAi Project at Harvard Medical School: Resources and Validation. Genetics 201, 843-852 (2015).

593 43. Filizola, M. \& Weinstein, H. The study of G-protein coupled receptor 594 oligomerization with computational modeling and bioinformatics. FEBS J 272, 2926-2938 (2005). 
596 44. Milligan, G. G protein-coupled receptor dimerisation: molecular basis and relevance to function. Biochim Biophys Acta 1768, 825-835 (2007).

598 45. Terrillon, S. \& Bouvier, M. Roles of G-protein-coupled receptor dimerization.

$599 \quad$ EMBO Rep 5, 30-34 (2004).

600 46. Yoshida, M., Oami, E., Wang, M., Ishiura, S. \& Suo, S. Nonredundant function of 601 two highly homologous octopamine receptors in food-deprivation-mediated signaling in Caenorhabditis elegans. J Neurosci Res 92, 671-678 (2014).

47. Lukasiewicz, S. et al. Hetero-dimerization of serotonin 5-HT(2A) and dopamine D(2) receptors. Biochim Biophys Acta 1803, 1347-1358 (2010).

48. Herrick-Davis, K. Functional significance of serotonin receptor dimerization. Exp Brain Res 230, 375-386 (2013).

49. Xie, Z., Lee, S.P., O'Dowd, B.F. \& George, S.R. Serotonin 5-HT1B and 5-HT1D receptors form homodimers when expressed alone and heterodimers when co-

50. Pellissier, L.P. et al. G protein activation by serotonin type 4 receptor dimers: evidence that turning on two protomers is more efficient. J Biol Chem 286, 99859997 (2011).

613 51. Renner, U. et al. Heterodimerization of serotonin receptors 5-HT1A and 5-HT7 differentially regulates receptor signalling and trafficking. J Cell Sci 125, 24862499 (2012).

616 52. Borroto-Escuela, D.O., Tarakanov, A.O. \& Fuxe, K. FGFR1-5-HT1A Depression. Trends Neurosci 39, 5-15 (2016). 
619 53. Moreno, J.L., Holloway, T., Albizu, L., Sealfon, S.C. \& Gonzalez-Maeso, J.

620

621

622

623

624

625

626

627

628

629

630

631

632

633

634

635

636

637

638

639

640

Metabotropic glutamate mGlu2 receptor is necessary for the pharmacological and behavioral effects induced by hallucinogenic 5-HT2A receptor agonists. Neurosci Lett 493, 76-79 (2011).

54. Saudou, F., Boschert, U., Amlaiky, N., Plassat, J.L. \& Hen, R. A family of Drosophila serotonin receptors with distinct intracellular signalling properties and expression patterns. EMBO J 11, 7-17 (1992).

55. Cvejic, S., Zhu, Z., Felice, S.J., Berman, Y. \& Huang, X.Y. The endogenous ligand Stunted of the GPCR Methuselah extends lifespan in Drosophila. Nat Cell Biol 6, 540-546 (2004).

56. Maqueira, B., Chatwin, H. \& Evans, P.D. Identification and characterization of a novel family of Drosophila beta-adrenergic-like octopamine G-protein coupled receptors. J Neurochem 94, 547-560 (2005).

57. van der Walt, S., Colbert, C., Varoquaux, G. The NumPy Array: A Structure for Efficient Numerical Vomputation. Computing in Science and Engineering, 22-30 (2011).

58. van der Walt, S., Schoenberger, J.L., Nunez-Iglesias, J., Boulogne, F., Warner, J.D., Yager, N., Gouillart, E., Yu, T., and the scikit-image contributors scikitimage: Image processing in Python. PerJ:e453 (2014).

59. Travis, E., Oliphant, E. A guide to NumPy. (2006).

60. Hunter, J.D. Matplotib: A 2D Graphics Environment. Computing in Science and Engineering, 90-95 (2007). https://archive.org/details/NumPyBook. 
641 61. Jones, E., Oliphant, E., Peterson, P., et al SciPy: Open Source Scientific Tools for Python. (2001-). https://www.scipy.org.

\section{Acknowledgements}

646 The authors dedicate this manuscript to Bruce Baker, who was one of the foremost

647 scientists in the field, and a great colleague and friend. The authors are grateful to

648 Richard Zoller, Yue Qian, Megan Aarnio, Heather Kudyba, Jacqueline Uribe,

649 Chidemman Ihenacho, Stefani Moore, Sampreet Reddy, Amanda Cameron, Chederli

650 Belongilot, Dylan Ricke, Kenneth Burgess, Amanda Redding, Erin Guillebeau, Jennifer

651 Murphy, Chantel McCarty, Sarah Murphy, Haley Grable, Mitch Hanson, Haein Kim, and

652 Sarah Rupert for technical assistance. We thank Bruce Baker, Carmen Robinett,

653 Edward Kravitz, Matthew Freeman, Mark Brown, Erika Matunis, Steve DiNardo,

654 Margaret Fuller, Alan Spradling, Hannele Rahuola-Baker, Eric Bohman, Celeste Berg,

655 Wolfgang Lukowitz, Patricia Moore, Jim Lauderdale, Michael Tiemeyr, Scott Dougan,

656 Rachel Roberts-Galbraith, and Rhonda Snook for helpful discussions, and Heath Aston

657 Zachary Letts for comments on the manuscript. We are especially grateful to Barry

658 Ganetzky for the $X \square X$, shi ${ }^{\text {ts }}$ fly stock and to Wolfgang Lukowitz for the use of his

659 microscope. This work was supported by NSF grants \#0841419 and \#1355009, and

660 UGA bridge funds given to CS.

661

\section{Author contributions}

663 M.M, B.B.P, and C.S developed and supervised the project, L.F.M coordinated the

664 mating experiments, K.K. identified the GPCRs expressed in testes tips and developed 
665 the computer analysis for the sperm counts, all authors performed the experiments,

666 M.M, K.K., and CS wrote the manuscript.

668 Competing interests

669 The authors declare no competing interests.

671 Tables

673 Table 1. MI ${ }^{\text {GSC }}$ from control, RNA-i and overexpression lines directed against G-

674 protein subunits and other signal transducers

675 UAS-driven expression for the listed genes in the germline via NG4-1. BL \#:

676 Bloomington stock number, Single and Mated: number of pHH3-positive GSCs/total

677 number of GSCs $=\mathrm{MI}^{\mathrm{GSC}}$, Diff: $\mathrm{MI}^{\mathrm{GSC}}$ of mated males minus $\mathrm{MI}^{\mathrm{GSC}}$ of non-mated males.

678 For RNA-i-lines marked by asterisks siblings outcrossed to wt did not show a strong

679 response to mating either, suggesting leakiness of the lines.

680

\begin{tabular}{|l|l|l|r|r|l|}
\hline Genotype & BL\# & Crossed to: & MI $^{\text {GSC }}$ Single & MI $^{\text {GiS }}$ Mated & Diff. \\
\hline UAS-G $G_{\alpha} f-i$ & 43201 & NG4-1 & $19 / 448=4.2 \%$ & $52 / 452=11.5 \%$ & 7.3 \\
\hline & $25930^{*}$ & NG4-1 & $54 / 1031=5.2 \%$ & $76 / 1183=6.1 \%$ & 0.9 \\
\hline UAS-G ${ }_{\alpha} i-i$ & 34924 & NG4-1 & $22 / 335=6.6 \%$ & $49 / 322=15.2 \%$ & 8.6 \\
\hline & 40890 & NG4-1 & $33 / 597=5.5 \%$ & $59 / 536=11 \%$ & 5.5 \\
\hline & 31133 & NG4-1 & $5 / 269=1.9 \%$ & $19 / 285=6.7 \%$ & 4.8 \\
\hline UAS-G $G_{\alpha} O-i$ & $34653^{*}$ & NG4-1 & $23 / 313=7.3 \%$ & $26 / 295=8.8 \%$ & 1.5 \\
\hline & 28010 & NG4-1 & $18 / 240=7.5 \%$ & $27 / 231=11.7 \%$ & 4.2 \\
\hline
\end{tabular}




\begin{tabular}{|c|c|c|c|c|c|}
\hline \multirow[t]{11}{*}{$U A S-G_{\alpha} q-i$} & 36820 & NG4-1 & $24 / 403=6.0 \%$ & $32 / 268=11.9 \%$ & 5.9 \\
\hline & 33765 & NG4-1 & $33 / 320=10 \%$ & $50 / 298=16.8 \%$ & 6.8 \\
\hline & 36775 & NG4-1 & $9 / 153=5.9 \%$ & $21 / 255=8.6 \%$ & 2.7 \\
\hline & 31268 & OR & $17 / 233=7.3$ & $15 / 169=8.9$ & 1.6 \\
\hline & & OR & $14 / 335=4.2$ & $12 / 164=7.3$ & 3.1 \\
\hline & & OR & $31 / 568=5.5$ & $27 / 333=8.1$ & 2.6 \\
\hline & & NG4-1 & $25 / 556=4.5$ & $8 / 296=2.7$ & -2.8 \\
\hline & & NG4-1 & $23 / 332=6.9$ & $23 / 291=7.9$ & 1 \\
\hline & & NG4-1 & $23 / 542=4.2$ & $19 / 577=3.3$ & -0.9 \\
\hline & & NG4-1 & $71 / 1430=5.0$ & $50 / 1164=4.3$ & -0.7 \\
\hline & 30735 & NG4-1 & $14 / 318=4.4 \%$ & $23 / 293=7.8 \%$ & 3.4 \\
\hline \multirow[t]{2}{*}{$U A S-G_{\alpha} S-i$} & 29576 & NG4-1 & $49 / 605=8.1 \%$ & $60 / 615=9.7 \%$ & 1.6 \\
\hline & 50704 & NG4-1 & $82 / 1137=7.3 \%$ & $121 / 1527=7.9 \%$ & 0.6 \\
\hline$U A S-G_{\beta} 5-i$ & 28310 & NG4-1 & $10 / 306=3.3 \%$ & $20 / 292=6.9 \%$ & 3.6 \\
\hline \multirow[t]{2}{*}{$U A S-G_{\beta} 13 F-i$} & 35041 & NG4-1 & $38 / 752=4.8 \%$ & $46 / 785=5.7 \%$ & 0.9 \\
\hline & 31134 & NG4-1 & $12 / 198=6 \%$ & $31 / 221=14 \%$ & 8.0 \\
\hline$U A S-G_{\beta} 76 C-i$ & 28507 & NG4-1 & $14 / 219=6.4 \%$ & $26 / 226=11.5 \%$ & 5.1 \\
\hline \multirow[t]{2}{*}{$U A S-G_{Y} 1-i$} & 25934 & NG4-1 & $21 / 283=7.4 \%$ & $45 / 311=14.4 \%$ & 7.0 \\
\hline & 34372 & NG4-1 & $21 / 434=4.8 \%$ & $46 / 400=11.5 \%$ & 6.7 \\
\hline \multirow[t]{2}{*}{$U A S-G_{\gamma} 30 A-i$} & 25932 & NG4-1 & $16 / 319=5.0 \%$ & $18 / 286=6.3 \%$ & 1.3 \\
\hline & 34484 & NG4-1 & $9 / 320=2.8 \%$ & $31 / 323=9.6 \%$ & 6.8 \\
\hline \multirow[t]{3}{*}{ UAS-CaMKI-i } & 41900 & NG4-1 & $17 / 337=5.0 \%$ & $31 / 328=9.4 \%$ & 4.4 \\
\hline & 35362 & NG4-1 & $10 / 222=4.5 \%$ & $17 / 212=8.0 \%$ & 3.5 \\
\hline & 26726 & NG4-1 & $16 / 301=5.3 \%$ & $22 / 282=7.8 \%$ & 2.5 \\
\hline \multirow[t]{2}{*}{ UAS-CaMKII-i } & 35330 & NG4-1 & $49 / 784=6.2 \%$ & $91 / 858=10.6 \%$ & 4.4 \\
\hline & 29401 & NG4-1 & $38 / 332=11.4 \%$ & $43 / 342=12.6 \%$ & 1.2 \\
\hline UAS-CrebA-i & 42526 & NG4-1 & $13 / 301=4.3 \%$ & $29 / 298=9.7 \%$ & 5.4 \\
\hline
\end{tabular}




\begin{tabular}{|c|c|c|c|c|c|}
\hline UAS-Gprk1-i & 35246 & NG4-1 & $13 / 323=4.0 \%$ & $15 / 207=7.4 \%$ & 3.4 \\
\hline & 28354 & NG4-1 & $13 / 304=4.3 \%$ & $24 / 289=12 \%$ & 7.7 \\
\hline \multirow[t]{2}{*}{ UAS-Gprk2-i } & 41933 & NG4-1 & $3 / 218=1.4 \%$ & $24 / 228=10.5 \%$ & 9.1 \\
\hline & 35326 & NG4-1 & $12 / 268=4.5 \%$ & $35 / 267=13.1 \%$ & 8.6 \\
\hline \multirow[t]{3}{*}{ UAS-IP3K-i } & 35296 & NG4-1 & $10 / 225=4.4 \%$ & $14 / 152=9.2 \%$ & 4.8 \\
\hline & 31733 & OR & $25 / 336=7.4 \%$ & $31 / 331=9.4 \%$ & 2.0 \\
\hline & 31733 & NG4-1 & $67 / 572=11.7 \%$ & $57 / 555=10.3 \%$ & -1.4 \\
\hline UAS-PKC53E-i & 34716 & NG4-1 & $18 / 304=5.9 \%$ & $22 / 289=7.6 \%$ & 1.7 \\
\hline \multirow[t]{5}{*}{ UAS-PKC98E-i } & 29311 & NG4-1 & $14 / 293=4.8 \%$ & $15 / 284=5.3 \%$ & 0.5 \\
\hline & 35275 & OR & $9 / 266=3.4 \%$ & $25 / 288=8.4 \%$ & 5.0 \\
\hline & & NG4-1 & $49 / 657=7.5 \%$ & $45 / 603=7.5 \%$ & 0.0 \\
\hline & 44074 & OR & $30 / 597=5.1$ & $36 / 346=10.4$ & 5.3 \\
\hline & & NG4-1 & $31 / 289=10.7$ & $38 / 318=11.9$ & 1.2 \\
\hline UAS-PLC2-i & 33719 & NG4-1 & $23 / 264=8.7 \%$ & $37 / 311=11.9 \%$ & 3.2 \\
\hline UAS-bsk-i & 53310 & NG4-1 & $21 / 380=5.5 \%$ & $32 / 346=9.3 \%$ & 3.8 \\
\hline UAS-Ira-i & 31595 & NG4-1 & $6 / 272=2.2 \%$ & $8 / 190=4.2 \%$ & 2.0 \\
\hline \multirow[t]{3}{*}{ UAS-kay-i } & 27722 & NG4-1 & $11 / 256=4.3 \%$ & $25 / 259=9.6 \%$ & 5.3 \\
\hline & 31322 & NG4-1 & $22 / 384=5.7 \%$ & $61 / 334=18.3 \%$ & 12.6 \\
\hline & 31391 & NG4-1 & $24 / 291=8.2 \%$ & $29 / 218-13.3 \%$ & 5.1 \\
\hline UAS-rl-i & 36059 & NG4-1 & $21 / 269=7.8 \%$ & $32 / 297=10.8 \%$ & 3.0 \\
\hline \multirow[t]{2}{*}{ UAS-wt-5-HT1A } & 27630 & NG4-1 & $16 / 335=4.8 \%$ & $30 / 302=10 \%$ & 5.2 \\
\hline & 27631 & NG4-1 & $10 / 240=4.2 \%$ & $24 / 271=8.9 \%$ & 4.7 \\
\hline \multirow[t]{2}{*}{ UAS-wt-CrebB17A } & 7220 & NG4-1 & $51 / 636=8.0 \%$ & $98 / 628=15.6 \%$ & 7.6 \\
\hline & 9232 & NG4-1 & $32 / 637=5.0 \%$ & $84 / 592=14.2 \%$ & 9.2 \\
\hline UAS-wt-CaMK2R3 & 29662 & NG4-1 & $20 / 292=6.8 \%$ & $28 / 289=10.0 \%$ & 3.2 \\
\hline UAS- CaMKII.T287A/ & 29663 & NG4-1 & $16 / 287=5.6 \%$ & $25 / 283=8.8 \%$ & 3.2 \\
\hline$U A S-w t-G \alpha S$ & 6489 & NG4-1 & $32 / 289=11.1 \%$ & $39 / 300=13.0 \%$ & 1.9 \\
\hline
\end{tabular}




\begin{tabular}{|l|l|l|r|r|l|}
\hline & 6489 & NG4-1 & $40 / 610=6.6 \%$ & $54 / 652=8.3 \%$ & 1.7 \\
\hline UAS-wt-Ira & 7216 & NG4-1 & $15 / 342=4.4 \%$ & $44 / 378=11.6 \%$ & 7.2 \\
\hline UAS-wt-Kay & 7213 & NG4-1 & $24 / 341=7.4 \%$ & $70 / 350=20.0 \%$ & 12.6 \\
\hline
\end{tabular}

\section{Table 2. MI ${ }^{\text {GSC }}$ from select RNA-i-lines directed against GPCRs}

685 UAS-driven expression of RNA-i for the listed GPCRs i via NG4-1 did not block the 686 increase in $\mathrm{MI}^{\mathrm{GSC}}$ in response to mating. $\mathrm{BL} \#$ : Bloomington stock number, Single and

687 Mated: number of pHH3-positive GSCs/total number of GSCs $=\mathrm{MI}^{\mathrm{GSC}}$, Diff: $\mathrm{MI}^{\mathrm{GSC}}$ of

688 mated males minus $\mathrm{MI}^{\mathrm{GSC}}$ of non-mated males. Note the variability in $\mathrm{MI}^{\mathrm{GSC}}$ among the 689 different genotypes. GPCRs marked by asterisks were excluded from further studies

690 because their siblings outcrossed to wt did not show a stronger response to mating than

691 the experimental (GPCR-i/NG4-1) flies.

\begin{tabular}{|l|l|r|r|c|}
\hline GPCR & BL \# & \multicolumn{1}{|c|}{ Single } & Mated & Diff. \\
\hline UAS-5-HT2A- $i$ & 31882 & $25 / 490=5.1$ & $36 / 465=7.3$ & 2.2 \\
\hline & 56870 & $19 / 582=3.3$ & $32 / 553=5.8$ & 2.5 \\
\hline UAS-5-HT2B-i & 60488 & $6 / 261=2.3$ & $21 / 272=7.7$ & 5.4 \\
\hline & 25874 & $4 / 228=1.7$ & $34 / 236=14.4$ & 12.7 \\
\hline UAS-Ado-R- $i$ & 27536 & $11 / 276=4.0$ & $20 / 209=9.6$ & 5.6 \\
\hline UAS-AKHR- $i$ & 29577 & $23 / 492=4.7$ & $54 / 574=9.4$ & 4.7 \\
\hline UAS-AR-2-i & 25935 & $13 / 363=3.6$ & $25 / 336=7.4$ & 3.6 \\
\hline UAS-CG13229- $i$ & 29519 & $9 / 285=3.2$ & $33 / 297=11.1$ & 7.9 \\
\hline UAS-CG14539-i $i$ & 25855 & $21 / 318=6.6$ & $31 / 307=10.1$ & 3.5 \\
\hline
\end{tabular}




\begin{tabular}{|c|c|c|c|c|}
\hline UAS-CG15556-i & 44574 & $28 / 425=6.6$ & $43 / 401=10.7$ & 4.1 \\
\hline \multirow[t]{2}{*}{ UAS-CG15744-i } & 28516 & $18 / 279=6.4$ & $27 / 252=10.7$ & 4.3 \\
\hline & 42497 & $23 / 323=7.1$ & $36 / 236=11.0$ & 3.9 \\
\hline UAS-CG30106-i & 27669 & $10 / 244=4.1$ & $34 / 273=12.4$ & 8.3 \\
\hline UAS-CG33639-i & 28614 & $32 / 300=10.7$ & $47 / 371=12.7$ & 2.0 \\
\hline UAS-CCHaR1-i* & 51168 & $27 / 407=6.6$ & $27 / 323=8.4$ & 1.8 \\
\hline UAS-Cry-i & 43217 & $43 / 389=11.0$ & $75 / 521=14.4$ & 3.4 \\
\hline UAS-CrzR-i & 52751 & $14 / 337=4.2$ & $31 / 333=9.3$ & 5.1 \\
\hline \multirow[t]{2}{*}{ UAS-Dop1R1-i } & 62193 & $12 / 352=3.4$ & $25 / 308=8.1$ & 4.6 \\
\hline & 55239 & $11 / 300=3.7$ & $44 / 267=16.5$ & 12.8 \\
\hline \multirow{3}{*}{$\begin{array}{l}\text { UAS-GABA } \\
B R 2-i\end{array}$} & 50608 & $6 / 176=3.4$ & $35 / 207=16.9$ & 13.5 \\
\hline & & & & \\
\hline & 27699 & $7 / 291=2.4$ & $18 / 282=6.4$ & 4.0 \\
\hline $\begin{array}{l}\text { UAS-GABA } \\
\text { BR3-i }\end{array}$ & 42725 & $10 / 190=5.3$ & $28 / 243=11.5$ & 6.2 \\
\hline UAS-Moody-i & 36821 & $5 / 301=1.7$ & $16 / 234=6.8$ & 5.1 \\
\hline UAS-Mth-I1-i & 41930 & $11 / 279=4.0$ & $42 / 300=14.0$ & 10 \\
\hline \multirow[t]{2}{*}{ UAS-Mth-I3-i } & 41877 & $54 / 817=6.6$ & $81 / 850=11.8$ & 5.2 \\
\hline & 36822 & $19 / 231=8.2$ & $39 / 241=16.2$ & 8.0 \\
\hline UAS-Mth-I8-i & 36886 & $48 / 933=5.1$ & $70 / 915=7.6$ & 2.5 \\
\hline UAS-Mth-I9-i & 51695 & $61 / 985=6.2$ & $82 / 910=9.0$ & 2.8 \\
\hline UAS-Mth-I15-i & 28017 & $14 / 349=4.0$ & $25 / 337=7.1$ & 3.1 \\
\hline UAS-PK1R-i & 27539 & $28 / 478=5.9$ & $37 / 375=9.9$ & 4.0 \\
\hline \multirow[t]{2}{*}{ UAS-Smo-i } & 27037 & $12 / 263=4.6$ & $33 / 288=11.4$ & 6.8 \\
\hline & 43134 & $19 / 274=7.0$ & $15 / 147=10.2$ & 3.2 \\
\hline UAS-Tre1-i & 34956 & $5 / 234=2.1$ & $28 / 252=11.1$ & 9.0 \\
\hline UAS-TKR86D-i* & 31884 & $31 / 564=5.5$ & $28 / 394=7.1$ & 1.6 \\
\hline
\end{tabular}




\begin{tabular}{|l|l|r|r|c|}
\hline UAS-TKR99D- $i$ & 55732 & $30 / 506=5.9$ & $49 / 467=10.5$ & 4.6 \\
\hline & 27513 & $4 / 240=1.7$ & $30 / 294=10.2$ & 8.5 \\
\hline
\end{tabular}

694 Table 3. MI ${ }^{\text {GSC }}$ from additional RNAi-lines with modified expression of the GPCRs 695 blocking the increase in $\mathrm{MI}^{\mathrm{GSC}}$ in mated males

696 BL \#: Bloomington stock number, Single and Mated: number of pHH3-positive

697 GSCs/total number of GSCs = $\mathrm{MI}^{\mathrm{GSC}}$, Diff: $\mathrm{MI}^{\mathrm{GSC}}$ of mated males minus $\mathrm{MI}^{\mathrm{GSC}}$ of non698 mated males.

\begin{tabular}{|l|l|r|r|c|}
\hline GPCR & BL \# & \multicolumn{1}{l|}{ Single } & Mated & Diff. \\
\hline $5 H T-1 A-i / N G 4-1$ & 25834 & $64 / 841=7.6$ & $67 / 777=8.6$ & 1 \\
\hline $5 H T-1 B-i / N G 4-1$ & 25833 & $16 / 256=6.2$ & $26 / 268=9.7$ & 3.5 \\
\hline & 27635 & $3 / 304=1.0$ & $27 / 317=8.5$ & 7.5 \\
\hline & 51842 & $20 / 381=5.2$ & $32 / 375-8.5$ & 3.3 \\
\hline $5 H T-7-$ i/NG4-1 & 54006 & $13 / 405=3.2$ & $23 / 351=6.5$ & 3.3 \\
\hline CG12290-i/NG4-1 & 32471 & $8 / 238=3.4$ & $17 / 229=7.4$ & 4.0 \\
\hline Mth-i/NG4-1 & 42520 & $4 / 260=1.5$ & $31 / 246=12.6$ & 11.1 \\
\hline
\end{tabular}

$701 \quad$ Figure legends

702

703 Figure 1. Mating increased male stem cell division frequency.

704 A) Cartoon depicting the stages of Drosophila spermatogenesis. Note that every GSC

705 division produces exactly 64 spermatids. GB: gonialblast, SG: spermatogonia, SC:

706 spermatocytes, SP: spermatids. 
707 A') The apical tip of a wt testis. The Faslll-positive hub (asterix) is surrounded by seven

708 Vasa-positive GSCs (green), one of which is in mitosis based on anti-pHH3-staining

709 (arrowhead). Scale bar: 10um.

710 B-F) Blue: non-mated condition, red: mated condition, ${ }^{\star *}$ : P-value $<0.001$, numbers of

711 GSCs and number of gonads $(\mathrm{n}=)$ as indicated.

712 B) Box plots showing the range of $\mathrm{MI}^{\mathrm{GSC}}$. Lines within boxes represent medians,

713 whiskers represent outliers.

$714 \mathrm{C}, \mathrm{D}) \mathrm{FDGs}$ showing bin of $\mathrm{MI}^{\mathrm{GSC}}$ (bin width=10) across a population of c) OR and D)

715 CS males on the $\mathrm{X}$-axis and the percentage of testes with each $\mathrm{MI}^{\mathrm{GSC}}$ on the $\mathrm{Y}$-axis.

716 E) Bar graph showing $\mathrm{SI}^{\mathrm{GSC}}$ of $O R$ males from three independent experiments.

717 F) Graph showing the percentage of EdU-marked OR GSCs on the Y-axis and hours of 718 feeding and mating on the X-axis.

720 Figure 2. Mating reduced the mature sperm pool.

721 A-C) Class 1,2 and 3 seminal vesicles from Mst35B-GFP males. Scale bars: $0.1 \mathrm{~mm}$;

722 arrows point to GFP-negative regions.

723 D-G) Numbers of seminal vesicles $(n=)$ as indicated, $n-m$ : non-mated, m: mated, ${ }^{* *}: P_{-}$

724 value $<0.001$

725 D-F) Bar graphs showing the distribution of Class 1 to 3 seminal vesicles in non-mated

726 and mated males at days one to three of the mating experiment. Three fly lines that

727 carry GFP-marked sperm were used: one that carries Dj-GFP (BL\#5417), one that

728 carries the Mst35B-GFP (BL\#58408), and one line that carries both constructs

729 (BL\#58406). 
G) Box plot showing sperm head volume (based on MST35B-GFP) per seminal vesicle

731 in non-mated and mated males on days one to three of the experiment.

\section{Figure 3. Mating did not affect GSC numbers.}

734 A-E) Blue: non-mated condition, red: mated condition, numbers of gonads $(n=)$ as

735 indicated, genotypes as indicated.

736 A-E) FDGs showing numbers of GSCs on the X-axis and percentage of testes with the

737 number of GSCs on the Y-axis. No difference in GSC numbers was observed between

738 non-mated and mated males from different genetic backgrounds.

740 Figure 4. G-proteins were required for the increase in $\mathrm{MI}^{\mathrm{GSC}}$ in response to

741 mating.

742 A) Cartoon depicting the activation of G-proteins upon GPCR stimulation by ligand. 1:

743 G-protein association before GPCR stimulation, 2: G-protein distribution after GPCR

744 stimulation, 3a-c: downstream signaling cascades. AC: Adenylyl Cyclase, cAMP: cyclic

745 Adenosine Monophosphate, PKA: Protein Kinase A, CREB: cAMP responsive element-

746 binding protein, PLC: Phospho Lipase C, DAG: Diacylglycerol, PKC: Protein Kinase C,

747 MAPK: Map Kinase, IP3: Inositol Triphosphate, CaMK: Calcium ${ }^{2+}$ calmodulin-dependent 748 protein kinase.

749 B, C) Bar graphs showing MI ${ }^{\text {GSC }}$. Blue: non-mated condition, red: mated condition, ${ }^{* * *}$ :

750 P-value $<0.001$, numbers of GSCs as indicated, genotypes as indicated.

751 B) Control animals increased $\mathrm{MI}^{\mathrm{GSC}}$ in response to mating.

752 C) Males expressing $G_{\alpha} i-i$ or $d n G_{\gamma} 1$ in the germline did not increase $\mathrm{MI}^{\mathrm{GSC}}$ after mating. 
754 Figure 5. Expression of RNA-i against seven distinct GPCRs blocked the increase

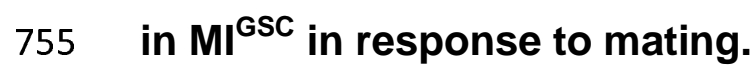

756 A-C) Bar graphs showing $\mathrm{MI}^{\mathrm{GSC}}$. Blue: non-mated condition, red: mated condition, ${ }^{* * *}$ :

757 P-value $<0.001$, numbers of GSCs as indicated, genotypes as indicated.

758 A) Control males have significantly higher $\mathrm{MI}^{\mathrm{GSC}}$ than their non-mated siblings.

759 B, C) Mated (B) GPCR-i/NG4-1 and (C) GPCR-i/NG4-2 males did not have significantly

760 higher $\mathrm{Ml}^{\mathrm{GSC}}$ compared to their non-mated siblings. 


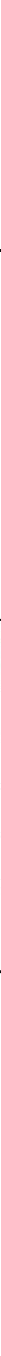


Mst35B-GFP class 1

Mst35B-GFP class 2
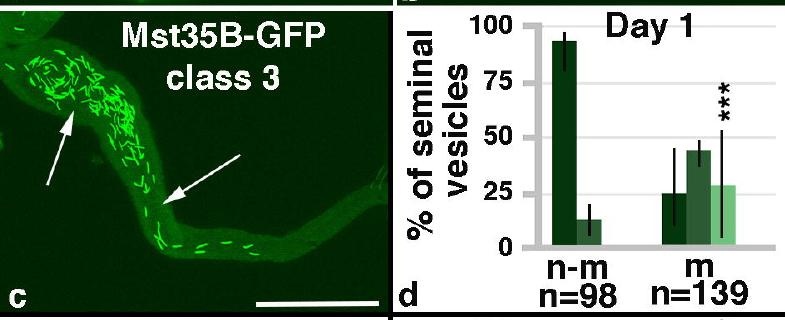

.

d

$\underbrace{n-m}_{n=98} n_{n=139}^{m}$

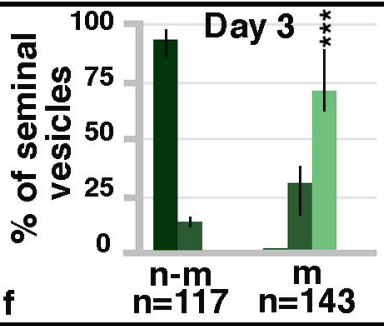

class 1

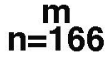

$n=117 \quad n=143$

class 2 class 3

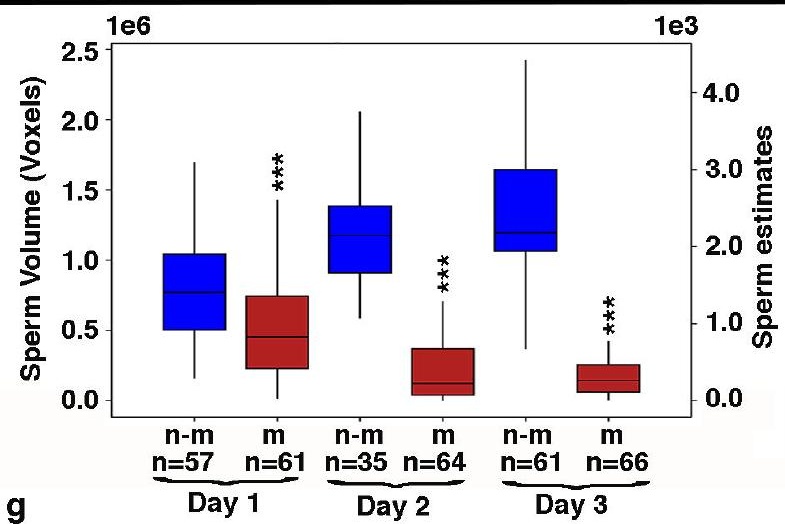



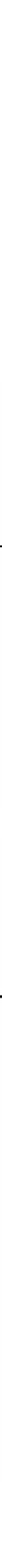